\title{
Random Mutagenesis by Insertion of Error-Prone PCR Products to the Chromosome of Bacillus subtilis
}

\author{
Bin Ye, Yu Li, Qing Tao, Xiaoliang Yao, Minggen Cheng and Xin Yan* \\ Key Laboratory of Agricultural Environmental Microbiology, Ministry of Agriculture, College of Life Sciences, Nanjing \\ Agricultural University, Nanjing, China
}

OPEN ACCESS

Edited by:

Kang Lan Tee,

The University of Sheffield,

United Kingdom

Reviewed by:

Tu Ran,

Tianjin Institute of Industrial

Biotechnology (CAS), China

Radivoje Prodanović,

University of Belgrade, Serbia

*Correspondence:

Xin Yan

yanxin@njau.edu.cn

Specialty section:

This article was submitted to

Microbial Physiology and Metabolism,

a section of the journal

Frontiers in Microbiology

Received: 07 June 2020

Accepted: 28 September 2020

Published: 13 November 2020

Citation:

Ye B, Li Y, Tao Q, Yao X, Cheng M and Yan X (2020) Random

Mutagenesis by Insertion

of Error-Prone PCR Products to the

Chromosome of Bacillus subtilis.

Front. Microbiol. 11:570280.

doi: 10.3389/fmicb.2020.570280
Bacillus subtilis is an attractive host for the directed evolution of the enzymes whose substrates cannot be transported across cell membrane. However, the generation of a mutant library in B. subtilis suffers problems of small library size, plasmid instability, and heterozygosity. Here, a large library of random mutant was created by inserting errorprone PCR (epPCR) products to the chromosome of $B$. subtilis. Specifically, the epPCR product was fused with flanking regions and antibiotic resistant marker using a PCRbased multimerization method, generating insertion construct. The epPCR product was integrated into the chromosome via homologous recombination after the insertion construct was transformed into the supercompetent cells of $B$. subtilis strain SCK6. The transformation efficiency of the insertion construct was improved through co-expressing homologous recombination-promoting protein $\mathrm{NgAgo}$, raising the number of competent cells, and increasing the length of flanking regions. A library containing $5.31 \times 10^{5}$ random mutants was constructed using per $\mu \mathrm{g}$ insertion construct, which is sufficient for directed evolution. The library generation process was accomplished within 1 day. The effectiveness of this method was confirmed by improving the activity of Methyl Parathion Hydrolase (MPH) toward chlorpyrifos and by enhancing the secretion level of MPH in B. subtilis. Taken together, the present work provides a fast and efficient method to integrate epPCR products into the chromosome of $B$. subtilis, facilitating directed evolution and expression optimization of target proteins.

Keywords: epPCR, chromosomal integration, large library, directed evolution, Bacillus subtilis, optimization of protein expression

\section{INTRODUCTION}

Directed evolution has been proved to be a powerful tool to improve the activity, stability, and substrate specificity of enzymes (Wong et al., 2004, 2007; Roodveldt et al., 2005; Romero and Arnold, 2009; Goldsmith and Tawfik, 2017; Zeymer and Hilvert, 2018). This tool involves two crucial steps: the generation of a library containing sufficient gene variants, and high-throughput screening of library members with desired properties (Wong et al., 2006; Packer and Liu, 2015). Because of its high transformation efficiencies, rapid growth rates, well-established manipulation 
approaches (Crameri et al., 1996), Escherichia coli is generally employed as the host organism for library creation. However, proteins are usually expressed in cytoplasm in E. coli, making the screening of the library more difficult if the substrate of the protein cannot be transported into the cell. Therefore, the organisms such as Bacillus subtilis and Pichia pastoris that can secret proteins into medium have been developed as alternative hosts for library generation (Reetz and Carballeira, 2007; Wang et al., 2012; Li Y. X. et al., 2017; Liu et al., 2017; Jiang et al., 2019).

B. subtilis is an important industrial host for the production of various recombinant proteins due to its GRAS (generally recognized as safe) status, excellent protein secretion ability and mature fermentation processes (Marcus et al., 2004; Terpe, 2006; Schumann, 2007; Yang et al., 2011; Commichau et al., 2014). Although B. subtilis has well-developed genetic manipulation tools, its transformation frequency is still much lower than that of E. coli. Thus, the library of random mutants generated through digestion and ligation is too small to fulfill the needs of directed evolution in B. subtilis. Several strategies have been adopted to address this limitation. A routine strategy is first constructing the library of variants in $E$. coli and then transferring the library members into B. subtilis, which is time-consuming and of low efficiency (You and Arnold, 1996; Zhao and Arnold, 1999; Caspers et al., 2010). Melnikov and Youngman (1999) attempted to clone the epPCR product via marker-replacement recombination with a structurally similar helper plasmid resident in the transformation recipient. But they found that it was difficult to recover $>10^{3}$ transformants/ $\mu \mathrm{g}$ of epPCR product. Given the phenomenon that multimeric plasmid has much higher (approximately three orders of magnitude) transformation frequency than that of the monomeric plasmid in B. subtilis (Canosi et al., 1978), Shafikhani et al. (1997) generated large libraries of random mutants $\left(3 \times 10^{6}\right)$ in B. subtilis by PCR-based plasmid multimerization method. In this method, epPCR product was fused with a linearized vector through PCR extension to generate linear plasmid multimer which could be converted to circular form through homologous recombination after entering the cell. However, each cell may take several variants, decreasing screening efficiency. Increasing the number of competent cells is an effective method to create a larger library. To this end, Zhang and Zhang (2011) constructed a B. subtilis strain SCK6 whose competence can be induced by controlling the expression of master regulator ComK artificially. The procedure for preparing the supercompetent cell of strain SCK6 was further improved by Li X. Z. et al. (2017). The transformation frequencies of multimeric plasmid, monomeric plasmid, and integration plasmid into strain SCK6 could reach $10^{7}, 10^{4}$, and $10^{5}$ transformants/ $\mu \mathrm{g}$ DNA, respectively.

This study aims to construct a large mutant library by inserting epPCR product into the chromosome of B. subtilis, which will solve the problems of plasmid instability and heterozygosity faced by multi-copy plasmid mediated strategies (Melnikov and Youngman, 1999; Zhang and Zhang, 2011; You et al., 2012). The library construction procedure was accomplished within 1 day and the generated library had sufficient mutants $\left(>10^{5}\right)$ for direct evolution.

\section{MATERIALS AND EQUIPMENT}

\section{Bacterial Strains, Primers, and Growth Conditions}

The B. subtilis SCK6 strain was provided by Dr. Daniel R. Zeigler from the Bacillus Genetic Stock Center (BGSC). The oligonucleotide synthesis and DNA sequencing were performed by Sangon Biotech Co., Ltd. (Shanghai, China). B. subtilis strains were cultivated in Lysogeny Broth (LB) (Bertani, 2004) medium, YN medium (Li X. Z. et al., 2017) or $2 \times$ SuperRich $(2 \times$ SR) (Song et al., 2017) medium. The LB medium consists of $1 \%$ tryptone, $0.5 \%$ yeast extract, and $0.5 \% \mathrm{NaCl}$. The YN medium is composed of $0.7 \%$ yeast extract and $1.8 \%$ nutrient broth. The $2 \times \mathrm{SR}$ medium (3\% tryptone, $5 \%$ yeast extract, and $0.6 \% \mathrm{~K}_{2} \mathrm{HPO}_{4}, \mathrm{pH} 7.2$ ) was used for fermentation. The solid medium was obtained by adding $15 \mathrm{~g} / \mathrm{L}$ agar to the liquid medium. Unless otherwise indicated, the final concentrations of antibiotics were as follows $(\mathrm{mg} / \mathrm{L})$ : zeocin (Zeo), 20; erythromycin (Em), 5; chloromycetin $(\mathrm{Cm})$, 5. The inoculums $(1 \%, \mathrm{~V} / \mathrm{V})$ were transferred into $250 \mathrm{~mL}$ flasks containing $30 \mathrm{~mL} 2 \times \mathrm{SR}$ medium and incubated at $37^{\circ} \mathrm{C}$ with shaking at $200 \mathrm{rpm}$. Chlorpyrifos (> 99\%) was purchased from the Macklin Biochemical Technology Co., Ltd. (Shanghai, China).

\section{Construction of $B$. subtilis SCK6A}

B. subtilis SCK6A was constructed by inserting the xyloseinducible promoter $P_{x y l A}$ controlled NgAgo-D663A-D738A (Fu et al., 2019) at the thrC locus in the chromosome of B. subtilis SCK6. The left flanking region (LF), $x y l R-P_{x y l A}$, and right flanking region (RF) were amplified from $B$. subtilis SCK6 genomic DNA using the primer pairs $\mathrm{P} 11 / \mathrm{P} 12$, P15/P16, and P19/P20, respectively. The $\mathrm{Cm}^{R}$ gene was amplified with the primer pair P13/P14 using plasmid pNW33N (BGSC) as the template. The NgAgo-D663A-D738A gene was amplified with the primer pair P17/P18 using plasmid pHT-XCR6 (MolecularCloud plasmid sharing platform Cat.no: MC_0068418) as the template. These five fragments were fused by overlap PCR in the following order: LF, $\mathrm{Cm}^{R}, x y l R-P_{x y l A}, N g A g o$, and RF. The PCR-product was directly transformed into strain SCK6, and the transformants were selected on LB plates containing $\mathrm{Cm}$.

\section{Construction of Methyl Parathion Hydrolase (MPH) Secretion Strain}

Four DNA fragments including $P_{c r y 3 A}$ promoter (Adams et al., $1994)$, the coding region of the signal peptide $\left(\mathrm{SP}_{\mathrm{AprE}}\right)$, the MPH-encoding gene mpd (Zhang et al., 2005), and the T1T2 transcription terminator (Hartl et al., 2001) were fused by overlap PCR (Shevchuk et al., 2004) using the primers listed in Table 1, generating the MPH expression cassette $P_{c r y 3 A}-m p d$. The expression cassette was then fused with flanking regions of $a m y E$ and Zeo resistant marker $\left(Z_{e o}{ }^{\mathrm{R}}\right)$, producing the insertion construct, which was then transformed into the competent cells of strain SCK6 and selected by Zeo. $P_{c r y 3 A}-m p d$ was finally integrated at the locus of $a m y E$ in the chromosome of strain SCK6, generating strain BPC1. 
TABLE 1 | Primers used in this study.

\begin{tabular}{|c|c|c|}
\hline Primers & Sequence $\left(5^{\prime}-3^{\prime}\right)$ & Purpose \\
\hline P1 & tgaactttatctgagaatagtcaatcttcggaaatcccaggtggc & For the construction of multimer of insertion construct (LF-Ab $\left.{ }^{R}-\mathrm{GOI}-\mathrm{RF}\right)$ \\
\hline P2 & catttttcttcctccctttcttatcataatacataattttcaaactg & \\
\hline P3 & cagtttgaaaattatgtattatgataagaaagggaggaagaaaaatg & \\
\hline P4 & gccacctgggatttccgaagattgactattctcagataaagttca & \\
\hline P5 & cagcgcaaatgctcccgctatcatcgagctccagcatccttgcagtcttcatatg & \\
\hline P6 & catatgaagactgcaaggatgctggagctcgatgatagcgggagcatttgcgctg & \\
\hline P7 & tttggaaagcgaggga & For the construction of variant $\mathrm{T} 47 \mathrm{C}$ \\
\hline P8 & ctgaacgccatcgtaaagattgacgttaacgcaaacaacaaacttatc & \\
\hline P9 & gataagtttgttgtttgcgttaacgtcaatctttacgatggcgttcag & \\
\hline P10 & cgttggttgtatccgtgt & \\
\hline P11 & agccgacactgcttcctg & For the construction of strain SCK6A \\
\hline P12 & catcatctgtatgaatcaaatcgcggccttcaatgcggtaagggttg & \\
\hline P13 & caacccttaccgcattgaaggccgcgatttgattcatacagatgatg & \\
\hline P14 & cggcaaccgagcgttctgaaactcacattaattgcgttgcg & \\
\hline P15 & cgcaacgcaattaatgtgagtttcagaacgctcggttgccg & \\
\hline P16 & catggatcccacctcctttaattgggactagtttggaccatttgtc & \\
\hline P17 & gacaaatggtccaaactagtcccaattaaaggaggtgggatccatg & \\
\hline P18 & caaagccgcgcattttcggaaggccttagaggaatccgacattagactcgaac & \\
\hline P19 & gttcgagtctaatgtcggattcctctaaggccttccgaaaatgcgcggetttg & \\
\hline P20 & agaatcgttgggcctget & \\
\hline P21 & cgcacctgcggtgctgcagcctgagcagacatgttgctgaacgcc & For the construction of variant G81T \\
\hline P22 & ggcgttcagcaacatgtctgctcaggctgcagcaccgcaggtgcg & \\
\hline P23 & gcggcggacttgccgtcgatgtcgagctgggtcgtgacgctggggtcgtc & For the construction of variant T806A \\
\hline P24 & gacgaccccagcgtcacgacccagctcgacatcgacggcaagtccgccgc & \\
\hline P25 & gccttcttgcgctccaccgcgacggacttgccgtcgatgtcgagc & For the construction of variant C821T \\
\hline P26 & gctcgacatcgacggcaagtccgtcgcggtggagcgcaagaaggc & \\
\hline P27 & gatgtggccgatgccggggaacgacaggtggctcgccgcgatcag & For the construction of variant C892T \\
\hline P28 & ctgatcgcggcgagccacctgtcgttccccggcatcggccacatc & \\
\hline P29 & gagtagttcaccggcacgaaatggtagcccttgccttcggcgc & For the construction of variant G938A \\
\hline P30 & gcgccgaaggcaagggctaccatttcgtgccggtgaactactc & \\
\hline
\end{tabular}

\section{Transformation of $B$. subtilis}

The B. subtilis SCK6 and SCK6A strains were inoculated into $4 \mathrm{~mL}$ of $\mathrm{YN}$ medium with $5 \mathrm{mg} / \mathrm{L}$ erythromycin in a test tube. The cells were cultivated at $37^{\circ} \mathrm{C}$ with shaking at $220 \mathrm{rpm}$ overnight $(\sim 12 \mathrm{~h})$. The culture was diluted to an optical density $\left(O D_{600 \mathrm{~nm}}=1.0\right)$ in a fresh YN medium containing $1.5 \%(\mathrm{w} / \mathrm{v})$ xylose and then was cultivated for $2 \mathrm{~h}$. The resulting cell cultures were the supercompetent cells that were transformed. $100 \mathrm{ng}$ of DNA was mixed with different volumes of the supercompetent cells in a 1.5 $\mathrm{mL}$ eppendorf tube and cultivated at $37^{\circ} \mathrm{C}$ with shaking at $220 \mathrm{rpm}$ for $90 \mathrm{~min}$.

\section{Screening of the Mutant Library}

The transformants were selected on LB plates containing Zeo and the colonies were then transferred to LB plates containing $50 \mathrm{mg} / \mathrm{L}$ chlorpyrifos using a sterile toothpick. Strain BPClwas used as the control. After incubation at $37^{\circ} \mathrm{C}$ for $12 \mathrm{~h}$, the $\mathrm{MPH}$ activity of each colony was determined according to the size of transparent halos. The mutants with larger transparent halos were selected for further verification.

\section{MPH Purification and Chlorpyrifos-Hydrolyzing Activity Assay}

The MPH purification was performed following the method described by Zhao et al. (2019). Briefly, the cells harboring MPH expression cassette were cultivated in a $2 \times$ SR medium for $36 \mathrm{~h}$ at $37^{\circ} \mathrm{C}$. The supernatant of the cultures was used for purification of the C-terminal His-tagged MPH through Ni-NTA affinity chromatography according to the manufacturer's instructions (Sangon Biotech Co., Ltd., Shanghai, China). Protein content was measured by the BCA Kit (Shanghai, Sangon Biotech Co., Ltd., China). The target proteins were analyzed by SDS-PAGE and the gel was stained with Coomassie brilliant blue R250. The activity of MPH toward chlorpyrifos was measured as described previously (Xie et al., 2014).

\section{Site-Directed Mutagenesis}

Each mutation was introduced into wildtype $P_{c r y 3 A}-m p d$ expression cassette through overlap PCR (Shevchuk et al., 2004). The genomic DNA of strain BPC1 was used as the template and the primers were listed in Table 1 . The PCR product was directly transformed into strain SCK6, generating the mutants. 


\section{METHODS}

A scheme summarizing the methodology for random mutagenesis by insertion of PCR products to the chromosome is illustrated in Figure 1. The epPCR product is firstly assembled into an insertion construct consisting of the left flanking region (LF), antibiotic resistant marker $\left(\mathrm{Ab}^{R}\right)$, epPCR product, and right flanking region (RF); then, after the insertion construct is transformed into $B$. subtilis competent cells, the epPCR product is inserted into the chromosome of $B$. subtilis through homologous recombination. The efficient assembly of the insertion construct is crucial in this method. The plasmid multimerization method (Shafikhani et al., 1997; You et al., 2012) is employed to assemble the insertion construct. First, LF, $\mathrm{RF}$, and $\mathrm{Ab}^{R}$ are fused through overlap PCR (Shevchuk et al., 2004) generating fragment RF-LF-Ab ${ }^{R}$; notably, RF is put ahead of LF and a cleavage site of restriction enzyme is introduced between RF and LF. The gene of interest (GOI) is amplified by epPCR. The left end of GOI overlapped (40-50 bp) with the right end of RF-LF- $A b^{R}$, while the right end of the GOI

\section{Chromosome}

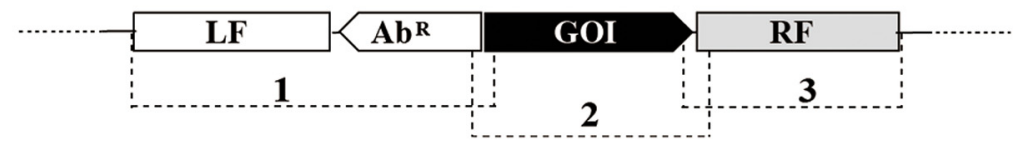

Fuse 3 and 1 by overlap PCR

Amplify 2 by error-prone PCR

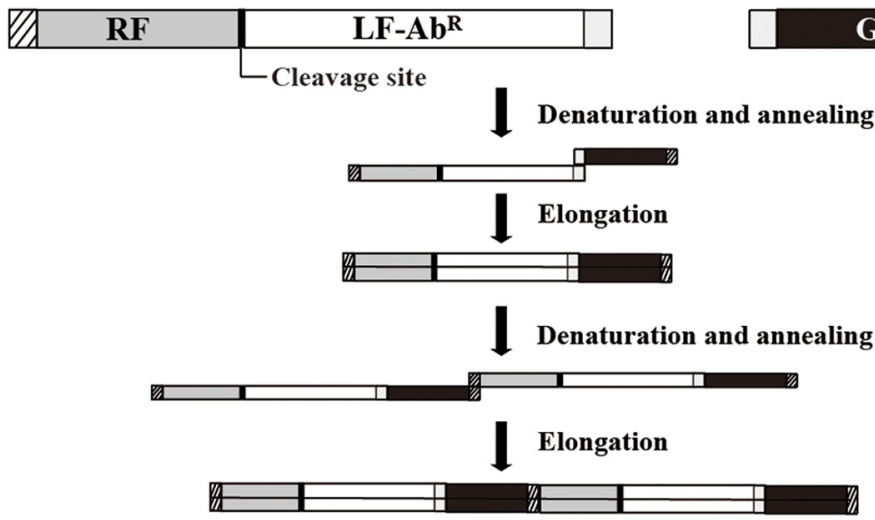

GOI
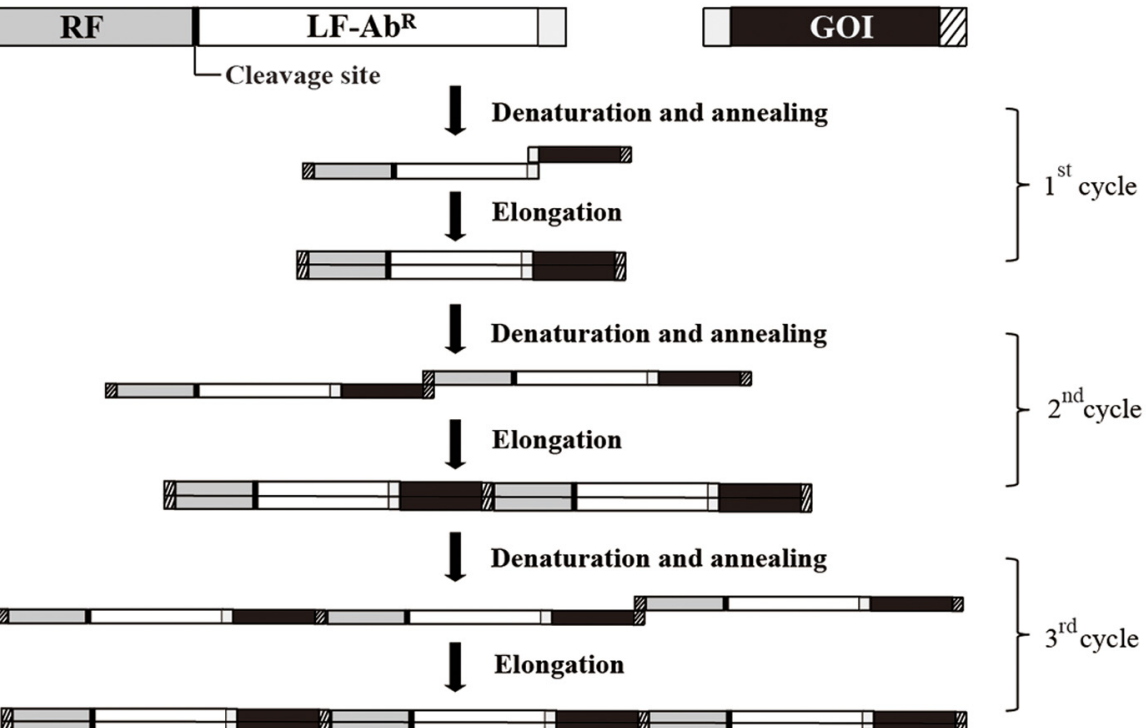

\section{Prolonged overlap extension PCR}

Multimers

Repeated denaturation, annealing and elongation

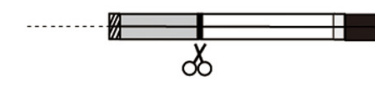

Monomer

Degestion

PCR

\section{Chromosome}
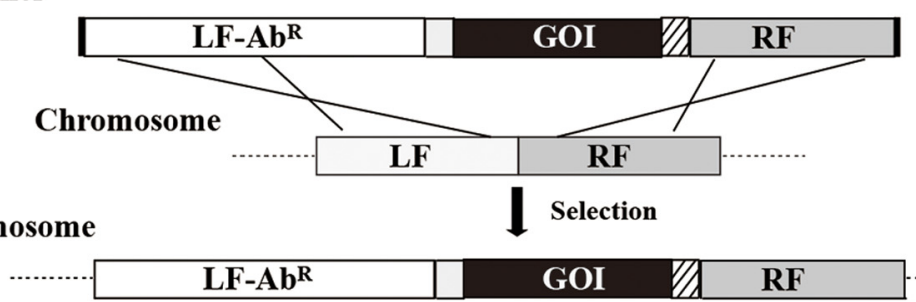

FIGURE 1 | The scheme for insertion of epPCR products to the chromosome of $B$. subtilis. Firstly, fragment RF-LF-Ab $R$ is generated by overlap PCR and fragment GOI is amplified by error-prone PCR (epPCR). Secondly, the DNA multimer is formed by prolonged overlap extension PCR using fragments RF-LF-Ab $R$ and GOI. Thirdly, the DNA multimers are digested to monomer (LF-Ab ${ }^{R}-\mathrm{GOI}-\mathrm{RF}$ ) at the cleavage site introduced between RF and LF. Finally, the insertion construct is transformed into competent cells. LF, left flanking region; $\mathrm{Ab}^{R}$, antibiotic resistant marker; GOI, gene of interest; RF, right flanking region; scissors, restriction endonuclease digestion. 
overlapped (40-50 bp) with the left end of RF-LF-Ab ${ }^{R}$. Then, the equal molar amount of GOI and RF-LF- $A b^{R}$ are mixed, and the multimer of insertion construct (LF-Ab ${ }^{R}-\mathrm{GOI}-\mathrm{RF}$ ) is generated by prolonged overlap extension PCR. Finally, the multimer is cut at the cleavage site between RF and LF, releasing the monomer of the insertion construct.

After the MPH mutant library was generated, following the above scheme, the RF of the amyE locus was amplified from the genomic DNA of strain BPC1 using primer pair P1/P5. Another fragment containing LF, $\mathrm{Zeo}^{R}$ and $P_{\text {cry } 3 A}$ was amplified as well using primer pair $\mathrm{P} 6 / \mathrm{P} 2$. These two fragments were fused by overlap PCR (Shevchuk et al., 2004) using primer pair P1/P2, resulting in fragment RF-LF-Zeo ${ }^{R}-P_{c r y 3 A}$. The cleavage site SacI was introduced between $\mathrm{RF}$ and $\mathrm{LF}$. The coding region of $\mathrm{SP}_{A p r E}$ and $\mathrm{MPH}$ was amplified by epPCR with primer pair P3/P4 using the Starmut Random Mutagenesis Kit (GenStar, Beijing, China). The PCR reaction solution with a total volume of 50 $\mu \mathrm{L}$ contained $0.02 \mathrm{ng} / \mu \mathrm{L}$ template, $25 \mu \mathrm{L} 2 \times$ buffer, $3 \mu \mathrm{L}$ StarMut Enhancer, $0.4 \mathrm{mM}$ primer pair P3/P4. The PCR was conducted as follows: initial denaturation at $95^{\circ} \mathrm{C}$ for $2 \mathrm{~min}$ and subsequent steps of denaturation at $94^{\circ} \mathrm{C}$ for $30 \mathrm{~s}$, annealing at $56^{\circ} \mathrm{C}$ for $1 \mathrm{~min}$, and extension at $72^{\circ} \mathrm{C}$ for $1 \mathrm{~min}$ for 30 cycles in total. The overlap of $47 \mathrm{bp}$ between the left end of the epPCR product and the right end of RF-LF-Zeo ${ }^{R}-P_{\text {cry } 3 A}$ was introduced through primers $\mathrm{P} 4$ and $\mathrm{P} 1$, and the overlap of $45 \mathrm{bp}$ between the right end of the epPCR product and the left end of RFLF-Zeo ${ }^{R}-P_{\text {cry } 3 A}$ was introduced through primers P3 and P2. To generate the multimer of fragment LF-Zeo ${ }^{R}-P_{c r y 3}$ - $m p d$-RF, each $50 \mu \mathrm{L}$ reaction system contained $0.2 \mathrm{mM}$ dNTP, an equal molar amount of RF-LF-Zeo ${ }^{R}-P_{\text {cry } 3 A}$ and epPCR product $(\sim 30 \mathrm{pmol}$

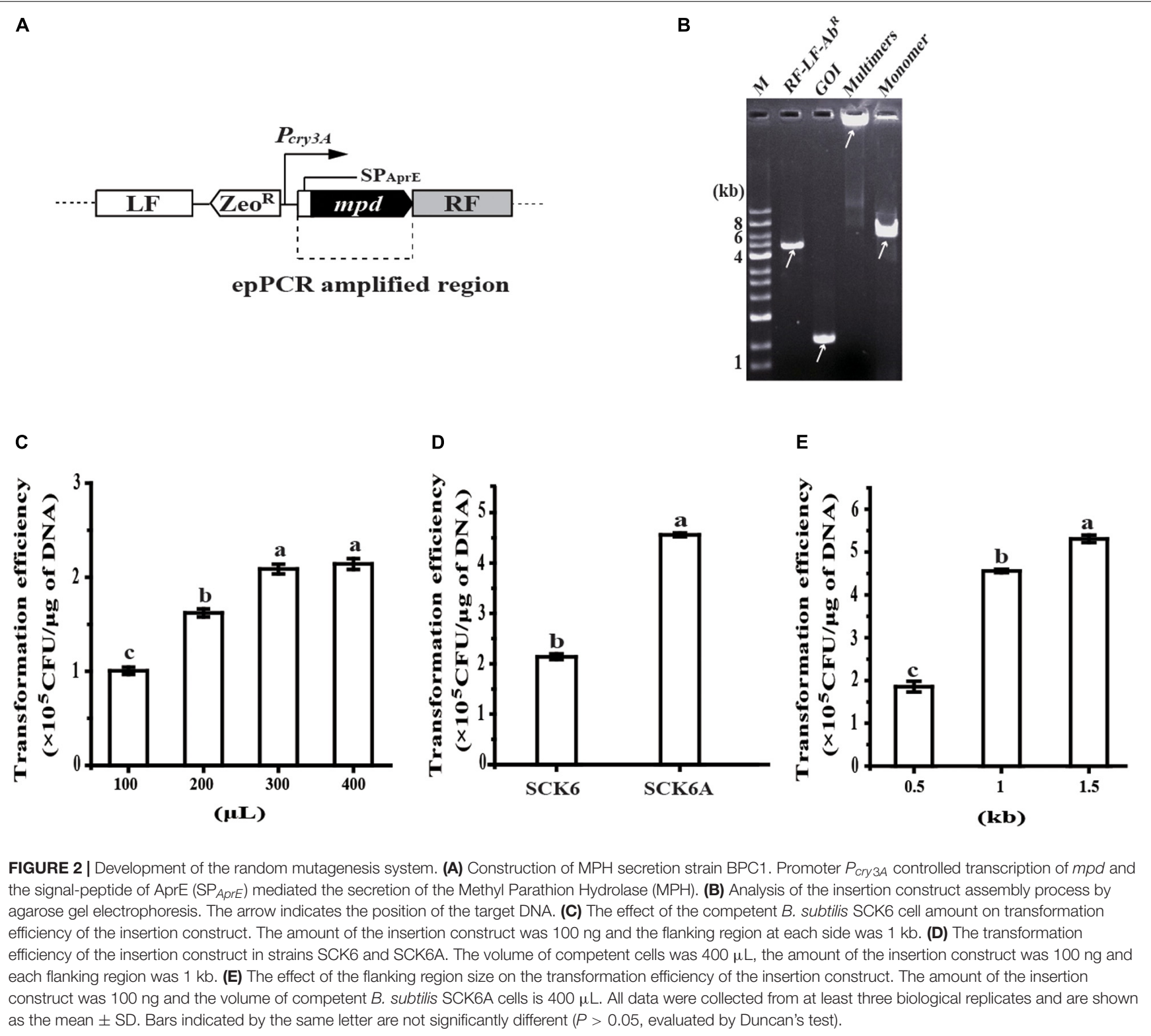


for each fragment), and $0.04 \mathrm{U} / \mu \mathrm{L}$ Phusion polymerase without the addition of primers. The prolonged overlap extension PCR was conducted as follows: denaturation at $98^{\circ} \mathrm{C}$ for $30 \mathrm{~s} ; 30$ cycles of denaturation at $98^{\circ} \mathrm{C}$ for $15 \mathrm{~s}$, annealing at $60^{\circ} \mathrm{C}$ for $15 \mathrm{~s}$, and extension at $72^{\circ} \mathrm{C}$ at a rate of $2 \mathrm{~kb} / \mathrm{min}$; followed by $72^{\circ} \mathrm{C}$ extension for $10 \mathrm{~min}$. The multimer product was digested with $\mathrm{SacI}$ to release the monomer of fragment LF-Zeo ${ }^{R}$ $P_{\text {cry } 3 \text { A-mpd-RF which was then transformed into the competent }}$ cells of strain SCK6.

\section{RESULTS}

\section{Development of the Random Mutagenesis System}

The random mutagenesis method was tested by the directed evolution of Methyl Parathion Hydrolase (MPH) to improve its activity toward chlorpyrifos, a pesticide contaminant that is often detected in food and the environment ( $\mathrm{Lu}$ et al., 2013). To begin with, the secretion expression of MPH was realized by inserting $\mathrm{MPH}$ expression cassette at the amyE locus in the chromosome of $B$. subtilis; the transcription of $m p d$ is driven by promoter $P_{c r y 3 A}$, and the secretion of $\mathrm{MPH}$ is mediated by signal-peptide of AprE ( $\left.\mathrm{SP}_{A p r E}\right)$ (Figure 2A). Then, the coding region of $\mathrm{SP}_{A p r E}$ and $\mathrm{MPH}$ is amplified by epPCR, and another fragment of RF-cleavage site (SacI)-LF$\mathrm{Zeo}^{R}-P_{\text {cry } 3 A}$ is generated via overlap PCR. After multimerization, the product was converted to a monomer of the insertion construct $\left(\mathrm{LF}-\mathrm{Zeo}{ }^{R}-P_{\text {cry } 3 A}-m p d-\mathrm{RF}\right)$ by SacI digestion, which was confirmed by agarose gel electrophoresis. As shown in Figure 2B, neither of the two fragments (RF-LF-Zeo ${ }^{R}-P_{\text {cry } 3 A}$ and epPCR product) could be detected on the gel after multimerization, indicating most of the two fragments were transformed into multimers. The SacI digestion product matched the size of insertion construct, which was further verified by DNA sequencing.

To enhance the transformation efficiency of the insertion construct, the amount of competent cells was first raised for each transformation reaction. Typically, when $400 \mu \mathrm{L}$ competent cells were mixed with $100 \mathrm{ng}$ DNA, the transformation efficiency reached $2.14 \times 10^{5} \mathrm{CFU} / \mu \mathrm{g} \mathrm{DNA}, 2.12$-fold than that of $100 \mu \mathrm{L}$ competent cells (Figure 2C). As protein NgAgo could enhance homologous recombination in bacteria (Fu et al., 2019; Wu et al., 2020), the gene encoding a mutant of $\mathrm{NgAgo}$ was then inserted into the chromosome of strain SCK6, under the control of xylose-inducible promoter $P_{x y l A}$. The transformation efficiency of the insertion construct in the NgAgo-expressing host (strain SCK6A) was about 2.13fold that in strain SCK6 (Figure 2D). We found that the transformation efficiency was positively correlated with the length of the flanking region. The flanking region of $0.5,1$, and $1.5 \mathrm{~kb}$ on each side led to the transformation efficiency of $1.85 \times 10^{5}, 4.56 \times 10^{5}$, and $5.31 \times 10^{5} \mathrm{CFU} / \mu \mathrm{g}$ DNA (Figure 2E), respectively.

\section{Screening of the Mutant Library of MPH Variants}

The flanking region of $1-\mathrm{kb}$ at each side of the insertion construct was used to generate a library of $m p d$ variants using strain SCK6A. Ten clones were randomly selected, and the DNA sequencing result of the $\mathrm{SP}_{A p r E}$ and $\mathrm{MPH}$ coding region shows that each mutant harbored 1-5 mutations, with an overall mutation rate of $0.31 \%$. As shown in Figure $3 \mathbf{A}$, the mutants were grown on an LB plate containing $50 \mathrm{mg} / \mathrm{L}$ chlorpyrifos. A transparent halo formed around the colony when chlorpyrifos was hydrolyzed by MPH (Xie et al., 2014). An enlarged halo indicated enhanced MPH activity, which may be caused by improved catalytic efficiency or increased MPH amount. Two mutants, named MT1 and MT2, which formed significantly larger transparent halos, were screened from about 12,000 colonies. When grown in a liquid medium, the growth curves of both mutants were similar to that of the parent strain BPC1 (data not shown), but the maximum extracellular MPH activity of mutants MT1 and MT2 were 2.47- and 2.77-fold that of strain BPC1 (Figure 3B), respectively.
A

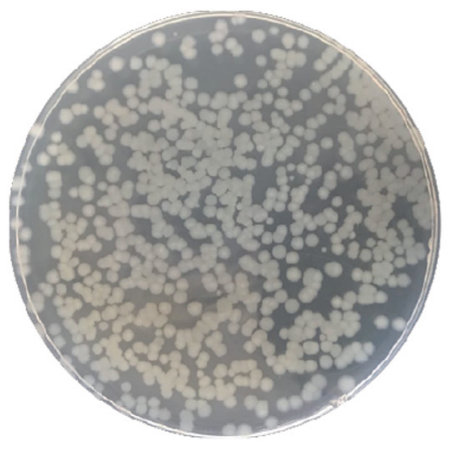

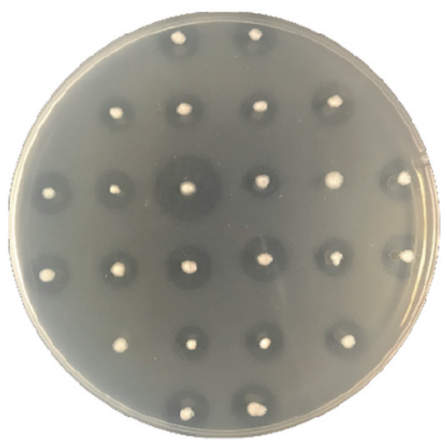

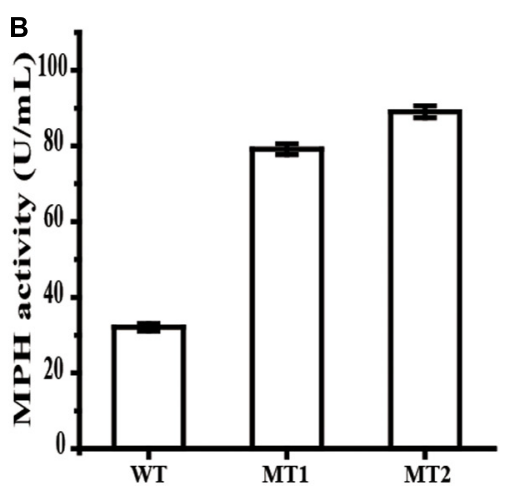

FIGURE 3 | Screening of the library of MPH variants. (A) Screening of the library on an LB plate containing $50 \mathrm{mg} / \mathrm{L}$ chlorpyrifos. Frist, transformants were selected on LB plates containing Zeocin. Then, the colonies were transferred to LB plates containing $50 \mathrm{mg} / \mathrm{L}$ chlorpyrifos using a sterile toothpick. When chlorpyrifos are hydrolyzed by MPH, a transparent halo forms around the colony. (B) The extracellular activities of MPH in the supernatant of strain BPC1 and its mutants. All data were collected from at least three biological replicates and are shown as the mean \pm SD. 


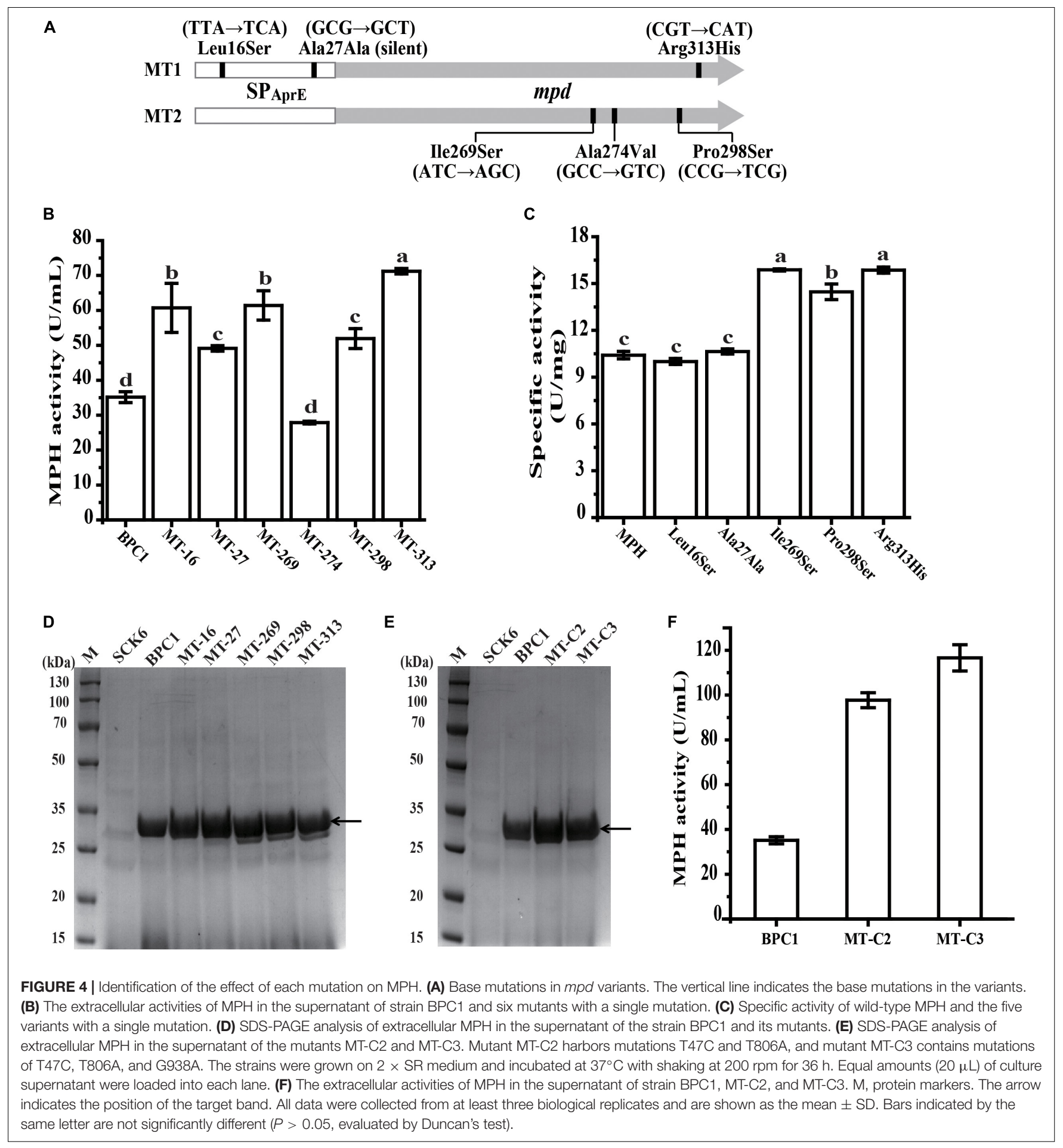

\section{Identification of the Effect of Each Mutation on MPH}

The $\mathrm{SP}_{\text {AprE }}$ and $\mathrm{MPH}$ coding region in mutant $\mathrm{MT} 1$ and MT2 were sequenced individually. The mutation sites are shown in Figure 4A. The mutant MT1 contained three mutations, with two (T47C and G81T) located in the $\mathrm{SP}_{\text {AprE }}$ coding region and one (G938A) located in
$\mathrm{MPH}$ coding region. Among these three mutations, two led to amino acid change (Leu16Ser and Arg313His), while G81T (Ala27Ala) is a synonymous mutation. All three mutations of mutant MT2 located in the MPH coding region (T806A, C821T, and $\mathrm{C} 892 \mathrm{~T})$, resulting in the amino acid substitutions of Ile269Ser, Ala274Val, and Pro298Ser, respectively. 
To determine the contribution of each mutation to the enhanced extracellular MPH activity in mutant MT1 and MT2, each mutation was individually introduced into strain BPC1, generating mutants of MT-16, MT-27, MT-269, MT-274, MT298, and MT-313. All six mutations did not affect the growth of their hosts compared with strain BPC1 (data not shown). The extracellular MPH activity of mutants MT-16, MT-27, MT269, MT-298, and MT-313 were 1.73-, 1.40-, 1.75-, 1.48-, and 2.03-fold that of strain BPC1, respectively, while mutant MT-274 exhibited a little bit lower extracellular MPH activity compared with strain BPC1 (Figure 4B). The extracellular MPH secreted by mutant MT-16, MT-27, MT-269, MT-298, and MT-313 were purified through Ni-NTA affinity chromatography individually. The MPH protein yield from mutant MT-269, MT-298, and MT-313 were close to that from strain BPC1, while the MPH protein yield from mutant MT-16 and MT-27 were 1.70- and 1.46-fold that from strain BPC1 (Figure 4D). Moreover, the specific activity of MPH variant T806A, C892T, and G938A were 1.53-, 1.43-, and 1.52-fold of wild-type $\mathrm{MPH}$, respectively, while the specific activity of MPH variant T47C and G81T was comparable with that of wild-type $\mathrm{MPH}$ (Figure 4C). Taken together, mutation $\mathrm{T} 47 \mathrm{C}$ and $\mathrm{G} 81 \mathrm{~T}$ in the $\mathrm{SP}_{\text {AprE }}$ coding region enhanced extracellular MPH activity through increasing $\mathrm{MPH}$ protein yield, while the contribution of mutation T806A, C892T, and G938A was enhancing the catalytic activity of MPH against chlorpyrifos. Additionally, to further elevate the extracellular $\mathrm{MPH}$ activity, two new mutants were created by recombining the mutations. Mutant MT-C2 harbors mutations T47C and T806A, and mutant MT-C3 contains mutations of T47C, T806A, and G938A. The extracellular MPH activities of mutant MTC2 and MT-C3 were 2.78- and 3.32-fold that of strain BPC1 (Figures 4E,F), respectively, indicating that the contribution of these mutations could be accumulated when combined.

\section{DISCUSSION}

In the present work, to efficiently insert epPCR products into the chromosome of B. subtilis, we first employed a PCR based multimerization method to fuse epPCR products with the flanking region and $\mathrm{Ab}^{R}$. The insertion construct was then transformed into supercompetent cells using a modified protocol, generating $5.31 \times 10^{5}$ transformations/ $\mu \mathrm{g}$ DNA. The library was created within 1 day and is large enough for the needs of directed evolution $\left(10^{4}-10^{5}\right.$ mutants are usually screened in directed evolution). Although the library generated here is smaller than those constructed by the multimeric plasmid method (Zhang et al., 2011; Li X. Z. et al., 2017), our method solves the problems of plasmid instability and heterozygosity encountered in the plasmid based method.

A crucial step in the present method is the assembly of the insertion construct (LF-Ab ${ }^{R}-$ GOI-RF). We attempted to fuse the three fragments (LF-Ab ${ }^{R}$, GOI, and RF) through PCR extension and Gibson assembly, but both methods resulted in a low yield of insertion construct. By contrast, the PCR based multimerization method (Shafikhani et al., 1997; You et al., 2012) realized the efficient assembly of the insertion construct (Figure 2B).
Moreover, we demonstrated that the transformation of the insertion construct could be substantially enhanced by raising the competent cell amount, expressing the recombinationpromoting protein, as well as increasing flanking region size (Figures 2C-E). NgAgo-like proteins were found to be able to improve homologous recombination by interacting with RecA in bacteria (Fu et al., 2019; Wu et al., 2020). The transformation of the insertion construct may be further optimized through testing different NgAgo-like proteins in future studies.

Plasmid or chromosome is used to carry the expression cassette when producing a protein in B. subtilis. The plasmid mediated protein expression has the limitations of plasmid instability and safety concern (the use of $\mathrm{Ab}^{R}$ ) (Bron et al., 1991), while the chromosomal integration manner can overcome the limitations of plasmid manner, but the low gene dose usually leads to a low protein yield. Therefore, the transcription and translation level of GOI needs to be dramatically enhanced to achieve high protein yield in an integrated manner. Mutant MT1 harbors two mutations in the $\mathrm{SP}_{\text {AprE }}$ coding region, both of which could improve the yield of MPH (Figures 4B,D). Nijland et al. (2007) have reported that a single amino acid change can remarkably increase the secretion level of $\beta$-toxin. Therefore, the random mutagenesis strategy established here could be applied in improving the protein secretion level in B. subtilis.

\section{DATA AVAILABILITY STATEMENT}

The raw data supporting the conclusions of this article will be made available by the authors, without undue reservation.

\section{AUTHOR CONTRIBUTIONS}

XYan developed the project idea and revised the manuscript. BY performed most of the experiments, analyzed data, and prepared the manuscript. YL, QT, and XYao did some data analysis and performed some experiments. MC provided consultation for the work and contributed significantly to the preparation of the manuscript. All authors reviewed and agreed on the content of the final manuscript.

\section{FUNDING}

This work was supported by the National Key R\&D Program of China 2019YFA0905200 and National Natural Science Foundation of China (31770125 and 31970099).

\section{ACKNOWLEDGMENTS}

We would like to acknowledge Dr. Daniel R. Zeigler from the BGSC for providing the B. subtilis SCK6 strain. This manuscript has been released as a pre-print at Authorea (Ye et al., 2020). 


\section{REFERENCES}

Adams, L. F., Mathewes, S., O'Hara, P., Petersen, A., and Gürtler, H. (1994). Elucidation of the mechanism of cryllIa overproduction in a mutagenized strain of Bacillus thuringiensis var. tenebrionis. Mol. Microbiol. 4, 381-389. doi: 10.1111/j.1365-2958.1994.tb01298.x

Bertani, G. (2004). Lysogeny at mid-twentieth century: P1, P2, and other experimental systems. J. Bacteriol. 186, 595-600. doi: 10.1128/JB.186.3.595-600. 2004

Bron, S., Holsappel, S., Venema, G., and Peeters, B. P. H. (1991). Plasmid deletion formation between short direct repeats in Bacillus subtilis is stimulated by single-stranded rolling-circle replication intermediates. Mol. Gen. Genet. 226, 88-96. doi: 10.1007/BF0027359

Canosi, U., Morelli, G., and Trautner, T. A. (1978). The relationship between molecular structure and transformation efficiency of some S. aureus plasmids isolated from B. subtilis. Mol. Gen. Genet. 166, 259-267. doi: 10.1007/ BF00267617

Caspers, M., Brockmeier, U., Degering, C., Eggert, T., and Freudl, R. (2010). Improvement of Sec-dependent secretion of a heterologous model protein in Bacillus subtilis by saturation mutagenesis of the $\mathrm{N}$-domain of the AmyE signal peptide. Appl. Microbiol. Biotechnol. 86, 1877-1885. doi: 10.1007/s00253-009$2405-\mathrm{x}$

Commichau, F. M., Alzinger, A., Sande, R., Bretzel, W., Meyer, F. M., Chevreux, B., et al. (2014). Overexpression of a non-native deoxyxylulose-dependent vitamin B6 pathway in Bacillus subtilis for the production of pyridoxine. Metab. Eng. 25, 38-49. doi: 10.1016/j.ymben.2014.06.007

Crameri, A., Whitehorn, E. A., Tate, E., and Stemmer, W. P. C. (1996). Improved green fluorescent protein by molecular evolution using DNA shuffling. Nat. Biotechnol. 14, 315-319. doi: 10.1038/nbt0396-315

Fu, L., Xie, C., Jin, Z., Tu, Z., Han, L., Jin, M., et al. (2019). The prokaryotic Argonaute proteins enhance homology sequence-directed recombination in bacteria. Nucleic. Acids. Res. 47, 3568-3579. doi: 10.1093/nar/ gkz040

Goldsmith, M., and Tawfik, D. S. (2017). Enzyme engineering: reaching the maximal catalytic efficiency peak. Curr. Opin. Struct. Biol. 47, 140-150. doi: 10.1016/j.sbi.2017.09.002

Hartl, B., Wehrl, W., Wiegert, T., Homuth, G., and Schumann, W. (2001). Development of a new integration site within the Bacillus subtilis chromosome and construction of compatible expression cassettes. J. Bacteriol. 183, 43934393. doi: 10.1128/JB.183.8.2696-2699.2001

Jiang, Z., Niu, T. F., Lv, X. Q., Liu, Y. F., Li, G. H., Lu, W., et al. (2019). Secretory expression fine-tuning and directed evolution of Diacetylchitobiose Deacetylase by Bacillus Subtilis. Appl. Environ. Microbiol. 85:e1076-19. doi: 10.1128/AEM. 01076- 19

Li, X. Z., Lu, Z. H., Zhou, Y. L., Li, S. Y., and Zhang, G. M. (2017). Preparation and transformation optimization for supercompetent B. subtilis SCK6 cells. Chin. J. Biotech. 33, 692-698. doi: 10.13345/j.cjb.160362

Li, Y. X., Yi, P., Yan, Q. J., Qin, Z., Liu, X. W., and Jiang, Z. Q. (2017). Directed evolution of a $\beta$-mannanase fromrhizomucor mieheito improve catalytic activity in acidic and thermophilic conditions. Biotechnol. Biofuels. 10:143. doi: 10.1186/s13068-017-0833-x

Liu, X., Liang, M., Liu, Y., and Fan, X. (2017). Directed evolution and secretory expression of a pyrethroid-hydrolyzing esterase with enhanced catalytic activity and thermostability. Microb. Cell Fact. 16:81. doi: 10.1186/s12934-0170698-5

Lu, P., Li, Q., Liu, H., Feng, Z., Yan, X., Hong, Q., et al. (2013). Biodegradation of chlorpyrifos and 3,5,6-trichloro-2-pyridinol by cupriavidus sp. dt-1. Bioresour. Technol. 127, 337-342. doi: 10.1016/j.biortech.2012.09.116

Marcus, S., Ajay, S., and Ward, O. P. (2004). Developments in the use of Bacillus species for industrial production. Can. J. Microbiol. 50, 1-17. doi: 10.1139/ w03-076

Melnikov, A., and Youngman, P. J. (1999). Random mutagenesis by recombinatorial capture of PCR products in Bacillus subtilis and Acinetobacter calcoaceticus. Nucleic. Acids. Res. 27, 1056-1062. doi: 10.1093/nar/27.4.1056

Nijland, R., Heerlien, R., Hamoen, L. W., and Kuipers, O. P. (2007). Changing a single amino acid in Clostridium perfringens beta-toxin affects the efficiency of heterologous secretion by Bacillus subtilis. Appl. Environ. Microbiol. 73, 1586-1593. doi: 10.1128/AEM.02356-06
Packer, M., and Liu, D. (2015). Methods for the directed evolution of proteins. Nat. Rev. Genet. 16, 379-394. doi: 10.1038/nrg3927

Reetz, M. T., and Carballeira, J. D. (2007). Iterative saturation mutagenesis (ISM) for rapid directed evolution of functional enzymes. Nat. Protoc. 2, 891-903. doi: 10.1038 /nprot.2007.72

Romero, P. A., and Arnold, F. H. (2009). Exploring protein fitness landscapes by directed evolution. Nat. Rev. Mol. Cell Biol. 10, 866-876. doi: 10.1038/nrm2805

Roodveldt, C., Aharoni, A., and Tawfik, D. S. (2005). Directed evolution of proteins for heterologous expression and stability. Curr. Opin. Struct. Biol. 15, 50-56. doi: $10.1016 /$ j.sbi.2005.01.001

Schumann, W. (2007). Production of recombinant proteins in Bacillus subtilis. Adv. Appl. Microbiol. 62, 137-189. doi: 10.1016/S0065-2164(07)62006-1

Shafikhani, S., Siegel, R. A., Ferrari, E., and Schellenberger, V. (1997). Generation of large libraries of random mutants in Bacillus subtilis by PCR-based plasmid multimerization. Biotechniques 23, 304-310. doi: 10.2144/97232rr01

Shevchuk, N. A., Bryksin, A. V., Nusinovich, Y. A., Cabello, F. C., Sutherland, M., and Ladisch, S. (2004). Construction of long DNA molecules using long PCRbased fusion of several fragments simultaneously. Nucleic. Acids. Res. 32:e19. doi: 10.1093/nar/gnh014

Song, Y., Fu, G., Dong, H., Li, J., Du, Y., and Zhang, D. (2017). High-Efficiency Secretion of beta-Mannanase in Bacillus subtilis through Protein Synthesis and Secretion Optimization. J. Agric. Food. Chem. 65, 2540-2548. doi: 10.1021/acs. jafc.6b05528

Terpe, K. (2006). Overview of bacterial expression systems for heterologous protein production: from molecular and biochemical fundamentals to commercial systems. Appl. Microbiol. Biot. 72, 211-222. doi: 10.1007/s00253-006-0465-8

Wang, X. J., Peng, Y. J., Zhang, L. Q., Li, A. N., and Li, C. (2012). Directed evolution and structural prediction of cellobiohydrolase II from the thermophilic fungus chaetomium thermophilum. Appl. Microbiol. Biotechnol. 95, 1469-1478. doi: 10.1007/s00253-011-3799-9

Wong, T. S., Roccatano, D., and Schwaneberg, U. (2007). Steering directed protein evolution: strategies to manage combinatorial complexity of mutant libraries. Environ. Microbiol. 9, 2645-2659. doi: 10.1111/j.1462-2920.2007.01411.x

Wong, T. S., Roccatano, D., Zacharias, M., and Schwaneberg, U. (2006). A statistical analysis of random mutagenesis methods used for directed protein evolution. J. Mol. Biol. 355, 858-871. doi: 10.1016/j.jmb.2005.10.082

Wong, T. S., Tee, K. L., Hauer, B., and Schwaneberg, U. (2004). Sequence saturation mutagenesis (SeSaM): a novel method for directed evolution. Nucleic. Acids. Res. 32:e26. doi: 10.1093/nar/gnh028

Wu, Y., Liu, Y., Lv, X., Li, J., and Liu, L. (2020). Camers-B: crispr/cpf1 assisted multiple-genes editing and regulation system for Bacillus subtilis. Biotechnol. Bioeng. 117, 1817-1825. doi: 10.1002/bit.27322

Xie, J., Zhao, Y., Zhang, H., Liu, Z., and Lu, Z. (2014). Improving methyl parathion hydrolase to enhance its chlorpyrifos-hydrolysing efficiency. Lett. Appl. Microbiol. 58, 53-59. doi: 10.1111/lam.12155

Yang, H., Liu, L., Li, J., Du, G., and Chen, J. (2011). Heterologous expression, biochemical characterization, and overproduction of alkaline alpha-amylase from Bacillus alcalophilus in Bacillus subtilis. Microb. Cell Fact. 10:77. doi: 10.1186/1475-2859-10-77

Ye, B., Li, Y., Tao, T., Yao, X. L., Cheng, M. G., and Yan, X. (2020). Random mutagenesis by insertion of error-prone PCR products to the chromosome of Bacillus subtilis. Authorea [Preprint]. doi: 10.22541/au.158955325.50010802

You, C., Zhang, X. Z., and Zhang, Y. H. P. (2012). Simple cloning via direct transformation of pcr product (DNA multimer) to Escherichia coli and Bacillus subtilis. Appl. Environ. Microbiol. 78, 1593-1595. doi: 10.1128/AEM. 07105-11

You, L., and Arnold, F. H. (1996). Directed evolution of subtilisin E in Bacillus subtilis to enhance total activity in aqueous dimethylformamide. Protein. Eng. 9, 77-83. doi: 10.1093/protein/9.1.77

Zeymer, C., and Hilvert, D. (2018). Directed evolution of protein catalysts. Annu. Rev. Biochem. 87, 131-157. doi: 10.1146/annurev-biochem-062917-012034

Zhang, X. Z., Cui, Z. L., Hong, Q., and Li, S. P. (2005). High-level expression and secretion of methyl parathion hydrolase in Bacillus subtilis WB800. Appl. Environ. Microbiol. 71, 4101-4103. doi: 10.1128/AEM.71.7.4101-4103.2005

Zhang, X. Z., Sathitsuksanoh, N., Zhu, Z., and Zhang, P. Y. H. (2011). One-step production of lactate from cellulose as the sole carbon source without any other organic nutrient by recombinant cellulolytic Bacillus subtilis. Metab. Eng. 13, 364-372. doi: 10.1016/j.ymben.2011.04.003 
Zhang, X. Z., and Zhang, Y. H. P. (2011). Simple, fast and high-efficiency transformation system for directed evolution of cellulase in Bacillus subtilis. Microb. Biotechnol. 4, 98-105. doi: 10.1111/j.1751-7915.2010.00230.x

Zhao, H., and Arnold, F. H. (1999). Directed evolution converts subtilisin E into a functional equivalent of thermitase. Protein. Eng. 12, 47-53. doi: 10.1093/ protein/12.1.47

Zhao, L. Z., Ye, B., Zhang, Q., Cheng, D., Zhou, C. Y., Cheng, S., et al. (2019). Construction of second generation protease-deficient hosts of Bacillus subtilis for secretion of foreign proteins. Biotechnol. Bioeng. 116, 2052-2060. doi: 10. 1002/bit.26992
Conflict of Interest: The authors declare that the research was conducted in the absence of any commercial or financial relationships that could be construed as a potential conflict of interest.

Copyright $\odot 2020 \mathrm{Ye}, \mathrm{Li}$, Tao, Yao, Cheng and Yan. This is an open-access article distributed under the terms of the Creative Commons Attribution License (CC BY). The use, distribution or reproduction in other forums is permitted, provided the original author(s) and the copyright owner(s) are credited and that the original publication in this journal is cited, in accordance with accepted academic practice. No use, distribution or reproduction is permitted which does not comply with these terms. 